\title{
Desafios na medição quantitativa da ingestão alimentar em estudos populacionais
}

\author{
Challenges in food intake assessment \\ in population studies
}

Luiz Antonio dos ANJOS 1,2

Danielle Ribeiro de SOUZA'

Sinara Laurini ROSSATO3

\section{RE S U M O}

A avaliação da ingestão alimentar em populações é uma medida cada vez mais presente em estudos epidemiológicos para a investigação da relação entre nutrição e doenças crônicas não transmissíveis já que as atuais recomendações indicam a necessidade de se manter uma vida ativa com controle da ingestão alimentar. A medida da ingestão alimentar quantitativa é geralmente feita por meio de recordatório (ou diário) alimentar de $24 \mathrm{~h}$ ou por questionários semiquantitativos de freqüência alimentar. O presente artigo discute os principais fatores que envolvem a obtenção e a análise dessas informações, particularmente no que diz respeito à ingestão energética. Fica evidente a necessidade de aprimorar as condições de obtenção das informações sobre as porções ingeridas, as tabelas de composição química de alimentos e da estimativa do gasto energético para a determinação das recomendações energéticas.

Termos de indexação: Ingestão de energia. Ingestão de alimentos. Metabolismo energético.

\section{A B S T R A C T}

The assessment of food intake in populations has been present in epidemiological studies involving the relationship between nutrition and non-communicable chronic diseases in accordance with the recommendation of maintenance of an active lifestyle with control of food intake. Quantitative food intake is measured via $24 \mathrm{~h}$ recall (or diary) or semi-quantitative food frequency questionnaires. The present paper discusses the main factors involved in obtaining and analyzing such information, particularly in relation to energy intake. It is evident that there should be better estimates of food portions, food composition tables, and energy expenditure to determine energy requirements.

Indexing terms: Energy intake. Eating. Energy metabolism.

\footnotetext{
${ }^{1}$ Universidade Federal Fluminense, Departamento de Nutrição Social, Laboratório de Avaliação Nutricional e Funcional. R. Mario Santos Braga, 30, Campus do Valonguinho, 24020-140, Niterói, RJ, Brasil. Correspondência para/Correspondence to: L. A. ANJOS.E-mail:<lanjos@gmail.com>.

2 Fundação Oswaldo Cruz, Escola Nacional de Saúde Pública Sergio Arouca. Rio de Janeiro, RJ, Brasil.

${ }^{3}$ Universidade do Vale do Rio dos Sinos, Programa de Pós-Graduação em Saúde Coletiva. São Leopoldo, RS, Brasil
} 


\section{N T R O D U Ç Ã O}

Com as mudanças no perfil epidemiológico e o aumento das doenças crônicas não transmissíveis (DCNT) cresceram investigações sobre a associação dessas doenças com o estilo de vida, ingestão alimentar, atividade física, tabagismo e ingestão de álcool ${ }^{1,2}$. Atualmente, diversos estudos relacionam as DCNT à alta ingestão de alimentos ricos em colesterol, ácidos graxos saturados e em outros lipídios, associada ao baixo consumo de fibras $^{2,3}$. Portanto, o conhecimento do perfil da ingestão alimentar na população é o método mais precoce que existe para identificar o risco de adoecer por DCNT em associação à nutrição. Como as recomendações atuais são de manter uma vida ativa com controle da ingestão alimentar ${ }^{4}$ existe a necessidade do desenvolvimento de instrumentos válidos para o diagnóstico de padrões alimentares inadequados na população em geral.

Os objetivos principais da avaliação da ingestão alimentar em populações são o cálculo de balanço (principalmente o energético); a identificação de padrões alimentares; a monitoração de tendências da ingestão de determinados (ou grupos de) alimentos; a identificação de segmentos da população com padrões alimentares associados a doenças; e o planejamento de programas de assistência alimentar. No processo de avaliação da ingestão alimentar existem, entretanto, desafios que devem ser enfrentados que vão desde como obter informações confiáveis, passando pela identificação de sub/super registros até o cálculo da energia e nutrientes com vistas ao estabelecimento de recomendações e intervenções. O presente artigo discute alguns desses desafios, particularmente no que se refere à ingestão energética (IE).

\section{Métodos de avaliação da ingestão, do consumo e da disponibilidade alimentar}

A disponibilidade alimentar de populações é principalmente avaliada por estudos de orçamentos familiares e pelas Folhas de Balanço
Alimentar (FBA). No Brasil tem-se, sistematicamente, realizado estudos do primeiro tipo, as Pesquisas de Orçamentos Familiares (POF). $\mathrm{O}$ pioneiro foi o Estudo Nacional da Despesa Familiar (ENDEF) em 1974-19755, seguido de POFs em 1986-1987, 1995, 2002-20036 e da atual, que se encontra em fase de coleta de dados (2008-09). As FBAs são compilações das contas nacionais sobre produção, importação, exportação e uso não alimentar de alimentos de um país e os dados são armazenados na Food and Agriculture Organization (FAO) para a realização de pesquisas mundiais de alimentação ${ }^{7}$. Os dados dessas pesquisas fornecem a disponibilidade de alimentos para a população e são, desta forma, considerados como estudos de consumo alimentar e não de ingestão alimentar.

A ingestão alimentar quantitativa individual pode ser estimada por diferentes métodos de inquérito alimentar. A escolha do método depende da população a ser estudada e do objetivo do estudo, ou seja, o tipo de informação dietética que se quer obter. Existem métodos recordatórios, nos quais os indivíduos recordam os alimentos já ingeridos e os métodos em que o indivíduo registra, no momento da ingestão, todos os alimentos ingeridos compondo, desta forma, um diário (registro) alimentar. Entre os primeiros, os mais importantes ${ }^{8}$ são o Questionário de Freqüência Alimentar (QFA) e o Recordatório de 24 horas (R24h).

O QFA tem sido descrito por diversos autores como o método mais adequado para identificar e descrever padrões alimentares em estudos epidemiológicos ${ }^{9}$. Este método inclui dois componentes básicos: a lista de alimentos e a freqüência com que estes alimentos foram ingeridos. Assim, os indivíduos indicam a freqüência de ingestão de um alimento em um determinado intervalo de tempo. Portanto, o desempenho de um QFA para avaliar a ingestão e o padrão alimentares depende da resposta a duas questões gerais ${ }^{10}:$ 1) Quão acurado pode ser o relato da freqüência de ingestão alimentar feita pelo indivíduo? e 2) Quão apropriada é a lista de alimentos? 
Em geral, o QFA não tem o objetivo de obter, de uma forma exata, a ingestão quantitativa de algum nutriente mas sim de identificar, sob a forma de triagem, grupos de indivíduos que apresentam ingestão inadequada de um determinado alimento para que se possa intervir, acompanhar, explorar com métodos quantitativos ou investigar associações a doenças ${ }^{11}$. Com a incorporação de porções dos alimentos é possível estimar a quantidade dos alimentos ingeridos em um determinado intervalo de tempo e o instrumento passa a ser chamado de QFA semi-quantitativo (QFA).

Várias estratégias são utilizadas para a construção da lista de alimentos. A extensão dessa lista deve ser avaliada, pois questionários muito extensos podem tornar a entrevista cansativa ${ }^{9} \mathrm{e}$ questionários muito curtos podem não representar adequadamente a ingestão alimentar ${ }^{12}$. Para a construção da lista dever-se-ia obter, preferencialmente, dados de uma amostra da população que será objeto de estudo. A lista seria, então, construída com base naqueles alimentos mais freqüentemente ingeridos e que mais contribuíssem para a ingestão total de energia ou dos nutrientes de interesse. Outras possibilidades incluem a aplicação de um QFA já validado em um estudo piloto, com posterior eliminação dos alimentos menos freqüentes ou que contribuem pouco para a variabilidade na ingestão dos nutrientes ${ }^{9}$, e a utilização de dados de estudos epidemiológicos que mostrem associações entre a ingestão de determinados alimentos e doenças específicas $^{9,10}$. Para que um item alimentar seja informativo ele deve ter três características gerais: o alimento deve ser ingerido com freqüência por um número importante de indivíduos; o alimento deve ser ingerido com distribuição diferente entre indivíduos e os alimentos devem contemplar os nutrientes de interesse ${ }^{9}$.

$\mathrm{Na}$ expectativa de obter instrumentos capazes de medir adequadamente a ingestão alimentar no Brasil, a construção, a adequação e a validação de QFA emergiu como uma importante área de estudo, originando diversos instrumentos validados direcionados a populações e a objetivos específicos. Aparentemente, o primeiro QFA que foi validado no Brasil foi proposto por Sichieri \& Everhart'13, baseando-se nas informações do ENDEF. Diversos autores adaptaram esse questionário e outros criaram as suas próprias listas baseando-se na ingestão alimentar em amostras de conveniência de indivíduos saudáveis ou com uma doença específica ${ }^{12,14-19}$. O Anexo apresenta uma coletânea dos estudos que validaram QFA no País.

Vale destacar dois estudos recentes, ambos de 2003, que construíram QFA baseando-se em dados de ingestão alimentar de amostra representativa de adultos nos municípios de São Paulo ${ }^{20}$ e de Niterói, RJ21, sendo, os primeiros QFA construídos baseando-se em amostra populacional que se tem notícia no País. Em ambos os casos, a lista foi construída com dados dietéticos de R24h de adultos (idade $\geq 20$ anos) em amostras de $1477 \mathrm{e}$ 1726 indivíduos em São Paulo e em Niterói, respectivamente. O QFA de São Paulo foi composto por uma lista de 59 alimentos para as mulheres, 60 para os homens e 60 para ambos os gêneros, com 4 opções de porção (pequena, média, grande e extra-grande). No caso de Niterói, a estratégia foi incluir na lista os alimentos que haviam sido ingeridos por, pelo menos, 30 indivíduos de modo que a versão final da mesma foi composta por 117 alimentos. Uma versão preliminar desse QFA já foi validada ${ }^{21}$.

O R24h consiste de uma entrevista na qual se obtém informação sobre todos os alimentos ingeridos nas 24 horas anteriores. O método é apropriado para a avaliação da ingestão média de alimentos e nutrientes de um grande número de indivíduos, desde que: 1) a amostra seja representativa da população e 2) os dias da semana sejam representados adequadamente ${ }^{8}$. Trata-se de um método barato, fácil de ser aplicado (com o devido treinamento), rápido e que pode ser utilizado com analfabetos, o que o torna mais atrativo do que os diários (que necessitam que o indivíduo registre os alimentos ingeridos) em estudos populacionais. Quando realizado pela primeira vez, o R24h tem a vantagem de não alterar o padrão de 
ingestão. Independentemente do método escolhido, existem desafios que pesquisadores enfrentam, seja na hora da aplicação do método ou na hora da análise dos dados.

\section{Desafios}

Tanto o R24h quanto os QFA dependem da memória e da colaboração dos indivíduos, ou seja, é necessário acreditar/confiar nas informações. Uma vez identificado o alimento consumido, precisa-se da quantidade o mais aproximada possível do que realmente foi ingerido. Uma alternativa, que é pouco prática, seria a apresentação de modelos de medidas caseiras (talheres, pratos, xícaras) ou modelos de alimentos para a decisão na hora da coleta dos dados. Em geral, os QFA trazem uma medida fixa para cada alimento, muitas vezes expressos em porções, que são, a priori, identificadas como pequena, média ou grande, de preferência obtidas de algum estudo prévio das características de ingestão da população. Neste aspecto, a introdução de registros fotográficos pode facilitar a identificação mais aproximada da real porção ingerida ${ }^{22}$.

Como o R24h avalia a ingestão de um dia, espera-se que esse dia seja típico para o indivíduo. Este fato traz problemas na hora do cálculo da quantidade de nutrientes e energia ingeridos, já que nem todos os alimentos são cadastrados em programas computacionais ou nas tabelas de composição química de alimentos. Quando isto ocorre, é recomendável lançar mão de algumas alternativas, como a procura junto ao fabricante ou a restaurantes (a internet facilitou muito esta busca) ou a obtenção da receita para a produção da refeição. Entretanto, caso o objetivo do estudo seja identificar a ingestão de micronutrientes, a busca da composição nutricional em rótulos é pouco eficaz, já que a rotulagem obrigatória dos alimentos não traz informações sobre todos os micronutrientes. O mesmo se aplica a estudos mais detalhados sobre a ingestão de gorduras, como ácidos graxos trans, monoinsaturados e poli- -insaturados. Nestes casos, a alternativa é buscar alimentos com características similares em tabelas de composição nutricional que trazem informações completas.

Um momento crucial na hora do cálculo da ingestão é a escolha da tabela de composição química de alimentos a ser usada, pois elas podem apresentar variações que necessitam ser identificadas e controladas para obter estimativas de ingestão as mais próximas possível do real. Os principais fatores inerentes às tabelas que podem resultar em diferenças entre os dados avaliados são: a) descrição incorreta de alimentos e/ou fontes de valores nutricionais; b) amostragem inadequada; c) utilização de métodos analíticos impróprios; d) inconsistência na terminologia utilizada para expressar certos nutrientes; e e) variabilidade resultante de fatores genéticos, ambientais, de preparo e processamento ${ }^{8}$.

A tabela mais antiga em uso no Brasil, gerada pelo Instituto Brasileiro de Geografia e Estatística (IBGE) para uso nas análises do ENDEF5, já não contempla mais a evolução da diversidade alimentar da população brasileira dos últimos 30 anos. Apesar de também ser uma compilação como as outras disponíveis, a tabela do IBGE foi gerada com informações detalhadas do nome popular e do nome científico dos alimentos cujas informações foram obtidas diretamente de fornecedores. Por causa da sua defasagem no tempo, o Ministério da Saúde financiou um projeto para o desenvolvimento de uma tabela que contemplou a medição da composição química dos alimentos mais freqüentemente ingeridos no País, como forma de atualizar a tabela do Instituto Brasileiro de Geografia e Estatística.

O projeto, coordenado pelo Núcleo de Estudos e Pesquisas em Alimentação (NEPA) da Universidade Estadual de Campinas (Unicamp), foi uma iniciativa para oferecer dados de um expressivo número de nutrientes em alimentos nacionais e regionais obtidos por meio de amostragem representativa e de análises realizadas 
somente por laboratórios com competência analítica comprovada segundo critérios internacionais. O produto final atual, a Tabela Brasileira de Composição de Alimentos (TACO), em sua segunda versão, contém dados de macronutrientes (carboidratos, lipídios e proteínas), colesterol, ácidos graxos, minerais e vitaminas de 495 alimentos ${ }^{23}$. Mesmo assim, a TACO parece cobrir somente algo em torno de $60 \%$ a $70 \%$ dos alimentos de inquéritos alimentares em adultos.

Comparações entre programas informatizados de avaliação da ingestão alimentar documentam as diferenças entre os dados calculados por procedimentos diferentes. Por exemplo, a comparação entre os resultados da ingestão energética e de macronutrientes (proteína, glicídio e lipídio) de um registro alimentar de 73 universitárias, obtidos usando dois pacotes computacionais (Programa de Apoio à Nutrição - CISNUT; versão 2,5 e Dietwin ${ }^{\odot}$ Clínico - versão 2.0.45) indicou não haver diferença significante na gramatura de proteínas (diferença média de 1,56, desvio-padrão - DP=14,1g) e no total da IE diária (diferença de $28,1, D P=282,1 \mathrm{kcal}$ ) entre os programas $^{24}$. As diferenças entre os programas na gramatura de glicídio $(7,53, \mathrm{DP}=28,3 \mathrm{~g})$ e de lipídios $(5,33, \mathrm{DP}=17,1 \mathrm{~g})$ também foram pequenas, mas estatisticamente significantes. Apesar da não diferença nos valores médios, houve grande diferença individual entre os programas para todos os macronutrientes e energia: -40 a $62 \mathrm{~g}$ para a proteína; -77 a 133 g para glicídios; -48 a 134g para lipídios e -754 a 1436kcal de IE, o que fez com que o coeficiente de variação fosse bastante alto. Em análise da ingestão alimentar de 100 crianças de 6 a 30 meses, Salles-Costa et al. ${ }^{25}$ documentaram diferenças quanto ao consumo de energia, de macronutrientes, vitamina $C$ e retinol entre os programas Virtual Nutri e NutWin.

A experiência da avaliação da ingestão alimentar usando o R24h, realizada por nutricionistas treinados, em uma amostra de 1726 indivíduos em estudo de base populacional em Niterói (RJ) identificou uma série de problemas inesperados e alguns já esperados. Como o estudo foi realizado em todos os segmentos da população ${ }^{26}$, alguns alimentos relatados foram inusitados como, pescoço de porco, pastel de feijão com carne seca, empadão de creme de milho, sendo necessário substituí-los por algum outro alimento semelhante em sua composição ou escolher alguma receita e então prepará-los em laboratório para posterior obtenção da gramatura e da respectiva informação nutricional. Entre os problemas esperados estavam a ingestão de produtos de marca pouco conhecida, de fabricação caseira (pães, por exemplo) ou do comércio da região cujas características, ou mesmo receitas, eram difíceis de obter. Os campeões nesses aspectos foram pães e bolos, particularmente os com cobertura, e os diversos tipos de tortas e doces.

\section{Cálculo do balanço energético}

O balanço energético consiste da diferença entre a ingestão energética e o gasto energético (GE) e é uma informação cada vez mais importante em estudos populacionais que envolvam a avaliação da atividade física relacionada ao estado nutricional ${ }^{27}$. Particularmente em estudos nutricionais, o interesse está em determinar se a IE está dentro do recomendado. Para tanto é necessário identificar o quanto de energia a população deve ingerir. As recomendações energéticas (RE) são dadas como o nível de ingestão energética da população que: 1) manterá o Balanço Energético (BE), sustentando a dimensão (e a composição) corporal e o nível de atividade física compatível com boa saúde a longo prazo e 2) permitirá nível de atividade física economicamente necessário e socialmente desejável ${ }^{28}$. Para simplificar, a recomendação energética é baseada na multiplicação da taxa metabólica basal (TMB) pelo nível de atividade física (NAF). Desta forma, o RE=IE=GE=TMB $x$ NAF.

Como a taxa metabólica basal não é rotineiramente medida, foi sugerido o uso das equações de predição compiladas por Schofield ${ }^{29}$ e que são baseadas nas informações de massa corporal 
e específicas para faixas etárias em homens e mulheres $^{27}$. A inadequação destas equações de predição para segmentos da população brasileira já foi documentada ${ }^{30,31}$. Basicamente os dados apontam para uma superestimativa média, pelas equações de Schofield, de $20 \%$ no valor de TMB medido $^{32}$. Resta saber se o outro componente, o nível de atividade física, também se altera. No inquérito domiciliar realizado na população adulta de Niterói (RJ) ${ }^{33}$, encontrou-se valores de NAF de 1,75 e 1,70 para mulheres e homens, respectivamente, valores que ficaram dentro da faixa de NAF, compatível com estilo de vida moderado ou ativo $(1,70$ a 1,99) segundo a classificação de organismos internacionais ${ }^{28}$. Entretanto, o gasto energético médio (erro padrão) medido foi de $1987,1(22,9)$ e $2382,0(38,0) \mathrm{kcal} / \mathrm{dia}^{1}$ para mulheres e homens, respectivamente, valores que se aproximaram dos valores de GE calculados pelo método da FAO usando TMB estimada e o valor mínimo de NAF leve, ou seja, 1,4. Portanto, a não medição da TMB deve ser compensada no valor do NAF na hora de estimar o GE de populações. De fato, se fossem utilizados os valores de GE estimados para a população adulta de Niterói usando-se os valores de TMB estimados e o NAF mínimo moderado $(1,7)$, os valores seriam bastante maiores (2297,5, DP=14,5 e 2900,8, $D P=30,8 \mathrm{kcal} / \mathrm{dia}^{1}$ para mulheres e homens, respectivamente) o que iria comprometer substancialmente as análises de balanço energético para essa população.

Não há dados de ingestão alimentar individual da população brasileira. Existem dados de disponibilidade de alimentos (DE), e, conseqüentemente, de energia e macronutrientes, vindos das várias POFs. Da mesma forma, existem dados antropométricos da população brasileira de estu-

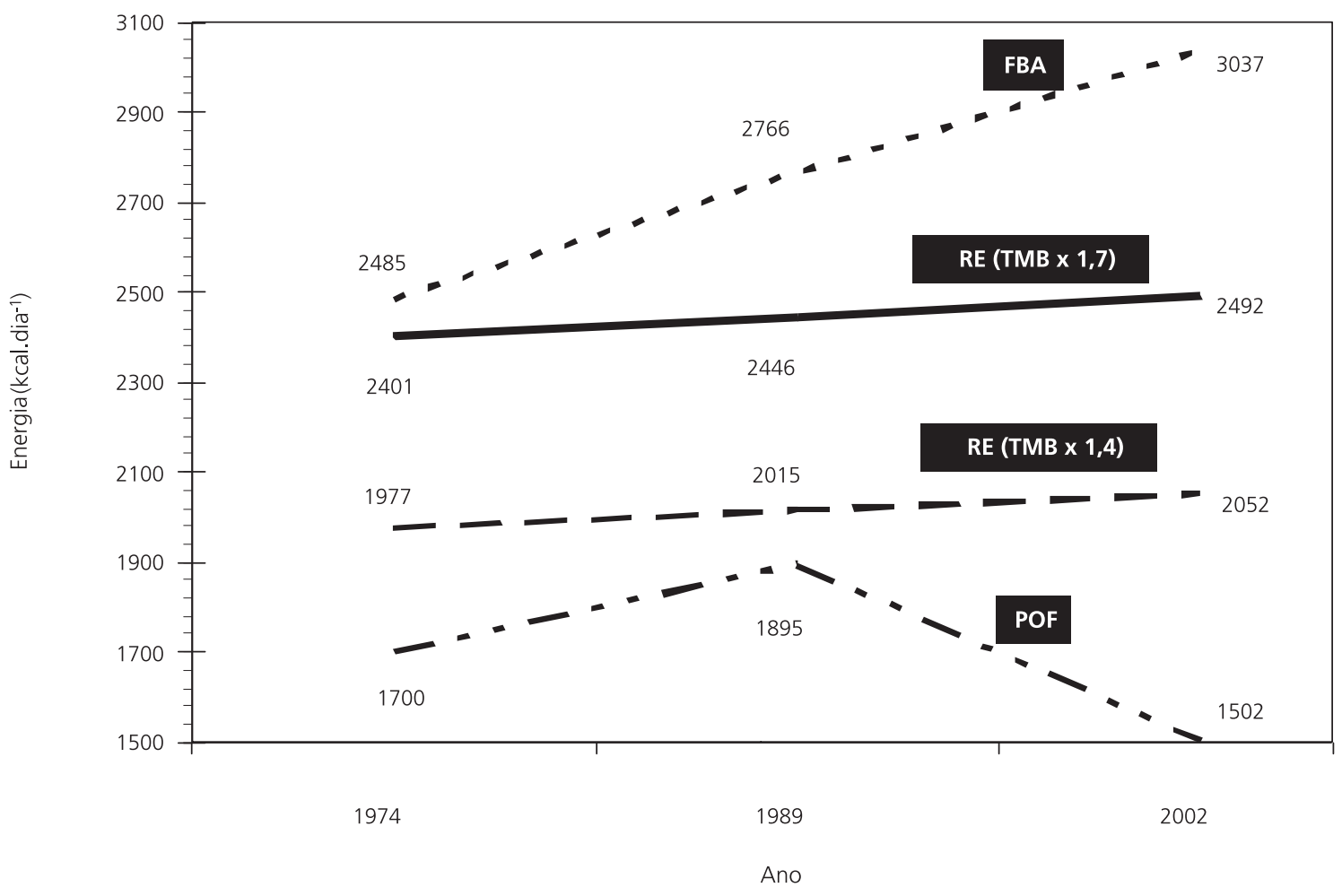

Figura 1. Evolução da disponibilidade energética pela Folha de Balanço Alimentar (FBA) e pelas Pesquisas de Orçamentos Familiares (POF) da população brasileira e a recomendação energética (RE) calculada pela TMB (usando a equação de Schofield ${ }^{28}$ ) e pelos valores mínimos de nível de atividade física sedentário $(1,4)$ e ativo $(1,7)$ na população adulta (idade $\geq 20$ anos) brasileira nos três inquéritos nacionais: ENDEF em 1974-1975, PNSN em 1989 e POF em 2002-2003. 
dos conduzidos nos últimos 30 anos (ENDEF em 1974-75; PNSN em 1989 e POF em 2002-2003). Desta forma, pode-se calcular as recomendações energéticas para a população brasileira para esses pontos no tempo que coincidem, aproximadamente, com os períodos da POF, para os quais há dados de disponibilidade energética. Para esses mesmos anos é possível obter a disponibilidade total de alimentos para a população brasileira a partir dos dados das folhas de balanço alimentar. A Figura 1 apresenta a comparação desses dados, sendo que os dados de RE foram calculados, usando as equações de Schofield ${ }^{29}$, somente para a população adulta (idade $\geq 20$ anos).

Ao assumir um valor fixo de nível de atividade física de 1,4 ou 1,7 (valores mínimos para estilo de vida sedentário e ativo, respectivamente ${ }^{28}$ ), fica evidente que a recomendação energética aumentou em um ritmo muito menor (e ficou sempre menor) do que a $D E$, segundo a folha de balanço alimentar, indicando balanço energético positivo, compatível com o aumento na prevalência de sobrepeso e obesidade observado na população brasileira no mesmo período ${ }^{34}$. Como não há dados sobre a evolução na realização de atividade física pela população brasileira e como os valores de NAF usados foram fixos, o aumento no RE veio exclusivamente do aumento da massa corporal da população. Os dados de DE da POF foram sempre muito baixos e, de forma inesperada, diminuiram na última pesquisa ${ }^{6}$. De fato, para contornar, em parte, os problemas de estimativa do consumo energético na POF, a atual POF (2008-2009) está realizando um registro alimentar em dois dias não consecutivos em associação ao questionário de despesa familiar ${ }^{35}$.

Como a estimação dos RE é dada pela estimação da TMB por meio dos dados de massa corporal, a superestimação dos RE torna-se muito crítica em indivíduos obesos. Nestes indivíduos, é freqüente a observação de BE negativo quando se estima o RE pelo método simplificado (TMB $x$ NAF) o que seria incompatível com o quadro nutricional que eles apresentam. Existem alguns problemas metodológicos básicos com a geração desses dados, o que pode comprometer sua inter- pretação. Em primeiro lugar, os estudos de ingestão alimentar em obesos parecem indicar que esse segmento da população subestima a ingestão energética sistematicamente ${ }^{36}$. Uma das possibilidades de identificar sub (ou super) registro consiste no cálculo da razão entre a IE e a TMB ${ }^{37}$. Dados da terceira fase do NHANES (Inquérito Nacional de Saúde e Nutrição) americano indicaram que essa razão decrescia com o aumento do IMC ${ }^{38}$, o que pode ser causado pelo menor valor do numerador (subestimativa da IE) e a superestimação do denominador (estimativa da TMB usando o valor de massa corporal nos obesos).

O gasto energético estimado pelo método fatorial detalhado (descrição de todas as atividades realizadas em um dia típico ou em um intervalo de 24 horas) parece ser superestimado, particularmente em mulheres, quando comparado ao GE medido ${ }^{39}$. A superestimação nos homens parece ser decorrente da superestimação da TMB enquanto nas mulheres a superestimação não pode ser explicada simplesmente pela TMB. O problema nas mulheres parece ser a dificuldade de detalhar o tipo e a duração das atividades domésticas, problema que é menor nos homens, pelo fato de eles não participarem dessas atividades com tanta freqüência. Na população adulta de Niterói, as tarefas domésticas representaram grande parcela do dia das mulheres (22\% do período em que permaneceram acordadas ou, aproximadamente, 3,5 horas), mas relativamente baixo (próximo a 1h) nos homens ${ }^{40}$.

Outro fator interveniente na estimativa do GE pelo método fatorial detalhado consiste na insuficiência de dados sobre o custo energético das atividades ${ }^{27,41}$. A publicação da FAONWHO/UNU ${ }^{28}$ lista as razões $\mathrm{GE} / \mathrm{TMB} /$ minuto de uma quantidade limitada de atividades. Em muitos casos, há descrição das atividades somente para homens. A outra alternativa constitui no chamado Compêndio de Atividades Físicas ${ }^{42}$, que traz uma lista extensa de atividades que são apresentadas como múltiplos do equivalente metabólico (MET) que, apesar de sua origem ainda incerta ${ }^{43}$, significa a energia de repouso (sentado) de um indivíduo 
adulto. Esta variável é usada universalmente como sendo de $3,5 \mathrm{~mL} \mathrm{O}^{2} / \mathrm{kg}$ massa corporal/minuto, mas existem evidências de que este valor é muito acima do medido em várias regiões do mundo ${ }^{41,44,45}$. Portanto, da mesma forma que para o NAF, é necessário saber se o custo energético das atividades expresso como múltiplos de MET presentes no Compêndio pode ser usado na população brasileira. No caso de caminhada no plano, esses valores parecem ser corretos $^{41}$.

Para a população em geral existe, ainda, a sugestão da eliminação dos dados muito baixos ou muito altos que seriam pré-estabelecidos, por exemplo, 500 e $4000 \mathrm{kcal} \mathrm{dia}^{-1}$, respectivamente ${ }^{9}$. Como os métodos de avaliação quantitativa da IE servem para grupos de indivíduos, não parece adequada a simples eliminação de um valor extremo, seja para mais ou para menos. Em um determinado dia, um segmento da população estará ingerindo muito mais (ou menos) do que o esperado ou habitual. Os casos típicos são a baixa ingestão associada a jejuns programados (por motivo religioso, por exemplo) ou não (caso de fome) ou a alta ingestão eventual causada, por exemplo, por participação em festas ou celebrações casuais. A eliminação sumária desses casos irá diminuir a variabilidade e camuflar casos de BE negativos e positivos.

É evidente a necessidade de desenvolver melhorias em vários dos procedimentos da determinação da ingestão alimentar de populações, particularmente no que se refere à obtenção da informação sobre o tamanho de porções, aos dados das tabelas de composição química dos alimentos e à estimação do gasto energético para a determinação das recomendações energéticas.

\section{A GRADECIMENTOS}

Parte dos dados apresentados vem de pesquisas que contaram com recursos do Conselho Nacional de Desenvolvimento Científico e Tecnológico (Processos 471172/2001-4, 475122/2003-8, 500672/2004-0, 361749/2004-0, 362105/2004-9), da Fundação
Oswaldo Cruz (PAPES III - Programa de Apoio a Projetos Estratégicos em Saúde - 250.139), da Coordenação de Aperfeiçoamento de Pessoal do Ensino Superior Programa Nacional de Cooperação Acadêmica (PROCAD) Processo 0257052. L.A. Anjos recebeu bolsa de produtividade em pesquisa do CNPq (Proces-sos 302952/2003-9, 311801/2006-4).

\section{OLA B OR A Ç Ã O}

L.A. ANJOS foi responsável pelas várias pesquisas cujos dados são apresentados, pelas análises dos dados apresentados na Figura e escreveu a primeira versão do manuscrito que foi revisado e aprovado pelos demais autores. D.R. SOUZA foi responsável pelas análises dos recordatórios de $24 \mathrm{~h}$ no inquérito domiciliar de Niterói. S.L ROSSATO realizou a revisão bibliográfica sobre os questionários de freqüência alimentar.

\section{REFERÊ NCIAS}

1. Popkin BM. The nutrition transition and obesity in the developing world. J Nutr. 2001;131:871S-3S.

2. World Health Organization. Diet, nutrition and prevention of chronic disease. World Health Org Tech Rep Ser. 916, 2003.

3. Schaefer EJ. Lipoproteins, nutrition, and heart disease. Am J Clin Nutr. 2002; 75:191-212.

4. World Health Organization. Process for a global strategy on diet, physical activity and health. Geneve: World Health Organization; 2003.

5. Instituto Brasileiro de Geografia e Estatística. Estudo Nacional da Despesa Familiar. Tabelas de composição dos alimentos. 4a.ed. Rio de Janeiro: IBGE; 1996.

6. Instituto Brasileiro de Geografia e Estatística. Pesquisa de Orçamentos Familiares 2002-2003. Análise da disponibilidade domiciliar de alimentos e do estado nutricional no Brasil. Rio de Janeiro: IBGE; 2004.

7. Food and Agriculture Organization. The Sixth World Food Survey. [cited 2009 Jan 5]. Available from: $<$ http://www.fao.org/ES/ESS/wfs.asp>.

8. Gibson RS. Principles of nutritional assessment. 2nd ed. New York: Oxford University Press; 2005.

9. Willett WC. Nutritional epidemiology. 2nd ed. New York: Oxford University Press; 1998.

10. Block G, Hartman AM, Dresser CM, Carroll MD, Gannon J, Gardner L. A data-based approach to 
diet questionnaire design and testing. Am J Epidemiol. 1986; 124:453-69.

11. Block G, Clifford C, Naughton MD, Henderson M, McAdams M. A brief dietary screen for high fat intake. J Nutr Edu. 1989; 21:199-207.

12. Teixeira MH, Veiga GV, Sichieri R. Avaliação de um questionário simplificado de freqüência de consumo alimentar como preditor de hipercolesterolemia em adolescentes. Arq Bras Cardiol. 2007; 88:66-71.

13. Sichieri RE, Everhart J. Validity of a Brazilian food frequency questionnaire against dietary recalls and estimated energy intake. Nutr Res. 1998; 18(10): 1649-59.

14. Cardoso M, Stocco R. Desenvolvimento de um questionário quantitativo de freqüência alimentar em imigrantes japoneses e seus descendentes residentes em São Paulo, Brasil. Cad Saúde Pública. 2000; 16:107-14.

15. Salvo VLA, Gimeno SGA. Reprodutibilidade e validade do questionário de freqüência de consumo de alimentos. Rev Saúde Pública. 2002; 36(4):505-12.

16. Furlan-Viebig R, Pastor-Valero M. Desenvolvimento de um questionário de freqüência alimentar para o estudo de dieta e doenças não transmissíveis. Rev Saúde Pública. 2004; 38:581-4.

17. Ribeiro AC, Sávio KEO, Rodrigues MLCF, Costa THMC, Schmitz BAS. Validação de um questionário de freqüência de consumo alimentar para a população adulta. Rev Nutr. 2006; 19(5):553-62.

18. Matarazzo HCZ, Marchioni DL, Figueiredo RAO, Slater B, Neto JE. Reprodutibilidade e validade do questionário de freqüência de consumo alimentar utilizado em estudo de caso-controle de câncer oral. Rev Bras Epidemiol. 2006; 9(3):316-24.

19. Queiróz AR, Costa CA, Popolim WD, Lima SCTC, Philippi ST. Avaliação do consumo alimentar pela Internet por meio de inquérito de freqüência dietética simplificado. Nutrire: Rev Soc Bras Alim Nutr. 2007; 32(1):11-22.

20. Fisberg RM, Colucci ACA, Morimoto JM, Marchioni DML. Questionário de freqüência alimentar para adultos com base em estudo populacional. Rev Saúde Pública. 2008; 42(3):550-4.

21. Zanolla AF, Olinto MTA, Henn RL, Wahrlich V, Anjos LA. Avaliação de reprodutibilidade e validade de um questionário de freqüência alimentar em adultos residentes em Porto Alegre/RS. Cad Saúde Pública. no prelo.

22. Zabotto CB, Viana RPT, Gil MF. Registro fotográfico para Inquéritos Dietéticos: utensílios e porções. Campinas: Unicamp; 1996.
23. Universidade Estadual de Campinas. Tabela brasileira de composição de alimentos. Versão II. 2.ed Campinas: Unicamp; 2006

24. Cruz CM, Anjos LA. Análise computacional de macronutrientes e energia de dietas de universitárias. Resumos do $5^{\circ}$ Congresso Nacional da Sociedade Brasileira de Alimentação e Nutrição. São Paulo, 1999. p.115.

25. Salles-Costa R, Antunes MML, Mello MA, Sichieri R. Comparação de dois programas computacionais utilizados na estimativa do consumo alimentar de crianças. Rev Bras Epidemol. 2007; 10(2):267-75.

26. Bossan FM, Anjos LA, Vasconcellos MTL, Wahrlich V. Nutritional status of the adult population in Niterói, Rio de Janeiro, Brazil: the nutrition, physical activity, and health survey. Cad Saúde Pública. 2007; 23(8):1867-76

27. Anjos LA, Wahrlich V. Gasto energético: medição e importância para a área de nutrição. In: Kac Gilberto, Sichieri R \& Gigante DP, organizadores, Epidemiologia nutricional. Rio de Janeiro: Atheneu; 2007. p.165-80.

28. Food and Agriculture Organization. Human Energy Requirements. Report of a Joint FAO/WHO/UNU Expert Consultation. Rome: FAO; 2004. Tech Rep Ser 1.

29. Schofield WN. Predicting basal metabolic rate, new standards and review of previous work. Hum Nutr: Clin Nutr. 1985; 39C(Suppl 1):5-41.

30. Cruz CM, Silva AF, Anjos LA. A taxa metabólica basal é superestimada pelas equações preditivas em universitárias do Rio de Janeiro, Brasil. Arch Latinoam Nutr. 1999; 49(3):232-7.

31. Wahrlich V, Anjos LA, Going SB, Lohman TG. Basal metabolic rate of Brazilians living in the Southwestern United States. Eur J Clin Nutr. 2007; 61(2):290-4.

32. Wahrlich V, Anjos LA, Vasconcellos MTL. Basal metabolic rate of Brazilian adults from a population based study: The nutrition, physical activity and health survey. [Em revisão].

33. Anjos LA, Ferreira BCM, Vasconcellos MTL, Wahrlich V. Gasto energético em adultos do Município de Niterói, Rio de Janeiro: resultados da pesquisa de nutrição, atividade física e saúde - PNAFS Cien Saúde Col. 2008; 13(6):1775-84.

34. Anjos LA. Obesidade e saúde pública. Rio de Janeiro: Fiocruz, 2006.

35. Sichieri R, Pereira RA, Martins A, Vasconcellos ABPA, Trichopoulou A. Rationale, design, and analysis of combined Brazilian household budget survey and food intake individual data. BMC Publ Health. 2008; 8:89. 
36. Black AE, Cole TJ. Within- and between-subject variation in energy expediture measured by the doubly-labelled water technique: implications for validating reported dietary energy intake. Eur J Clin Nutr. 2000; 54:386-94.

37. Goldberg GR, Black AE, Jebb AS, Cole TJ, Murgatroyd PR, Coward WA, Prentice AM. Critical evaluation of energy intake data using fundamental principles of energy intake physiology: 1. Derivation of cut-off limits to identify under-recording. Eur J Clin Nutr. 1991; 45(12):569-81

38. Briefel RB, Sempos CT, Mcdowell MA, Chien SC, Alaimo K. Dietary methods research in the third National Health and Nutrition Examination Survey: underrreporting of energy intake. Am J Clin Nutr. 1997; 65(Suppl):1203s-9s

39. Anjos LA, Vasconcellos MTL, Barbosa TB, Ferreira BCM, Machado JM, Wahrlich V. Time allocation or $24 \mathrm{~h}$ activity recall questionnaires overestimate energy expenditure in adults. A population-based study in Niterói, Rio de Janeiro, Brazil. [Em revisão].

40. Barbosa TBC, Anjos, LA, Vasconcellos MTL. Padrão de atividade física de adultos residentes no município de Niterói, Rio de Janeiro: Resultados da Pesquisa de Nutrição, Atividade Física e Saúde - PNAFS. [Em revisão].

41. Anjos LA, Wahrlich V, Bossan FM, Salies MN, Silva PB. Energy expenditure of walking at different intensities in Brazilian college women. Clin Nutr. 2008; 27(1):121-5.

42. Ainsworth BE, Haskell WL, Whitt MC, Irwin ML, Swartz AM, Strath SJ, et al. Compendium of physical activities: an update of activity codes and MET intensities. Med Sci Sports Exerc. 2000; 32(Suppl 9): S498-516.

43. Howley ET. You asked for it: question authority. ACSM Health Fitness J. 2000; 4:6-8.

44. Byrne NM, Hills AP, Hunter GR, Weinsie RL, Schutz Y. Metabolic equivalent: one size does not fit all. J Appl Physiol. 2005; 99:1112-9.

45. Gunn SM, van der Ploeg GE, Withers RT, Gore CJ, Owen N, Bauman AE, et al. Measurement and prediction of energy expenditure in males during household and garden tasks. Eur J Appl Physiol. 2004; 91:61-70.

46. Chiara VL, Sichieri R. Food consumption of adolescents. A simplified questionnaire for evaluating cardiovascular risk. Arq Bras Cardiol. 2001; 77:337-41.

47. Slater B, Philippi ST, Fisberg RM, Latorre MRDO. Validation of a semi-quantitative adolescent food frequency questionnaire applied at a public school in Sao Paulo, Brazil. Eur J Clin Nutr. 2003; 57(5): 629-35.

48. Colucci ACA, Philippi ST, Slater B. Desenvolvimento de um questionário de freqüência alimentar para avaliação do consumo alimentar de crianças de 2 a 5 anos de idade. Rev Bras Epidemiol. 2004; 7 : 393-401.

Recebido em: 8/1/2009

Versão final reapresentada em: 20/1/2009 Aprovado em: 12/2/2009 
DESAFIOS NA MEDIÇÃO DA INGESTÃO | 161

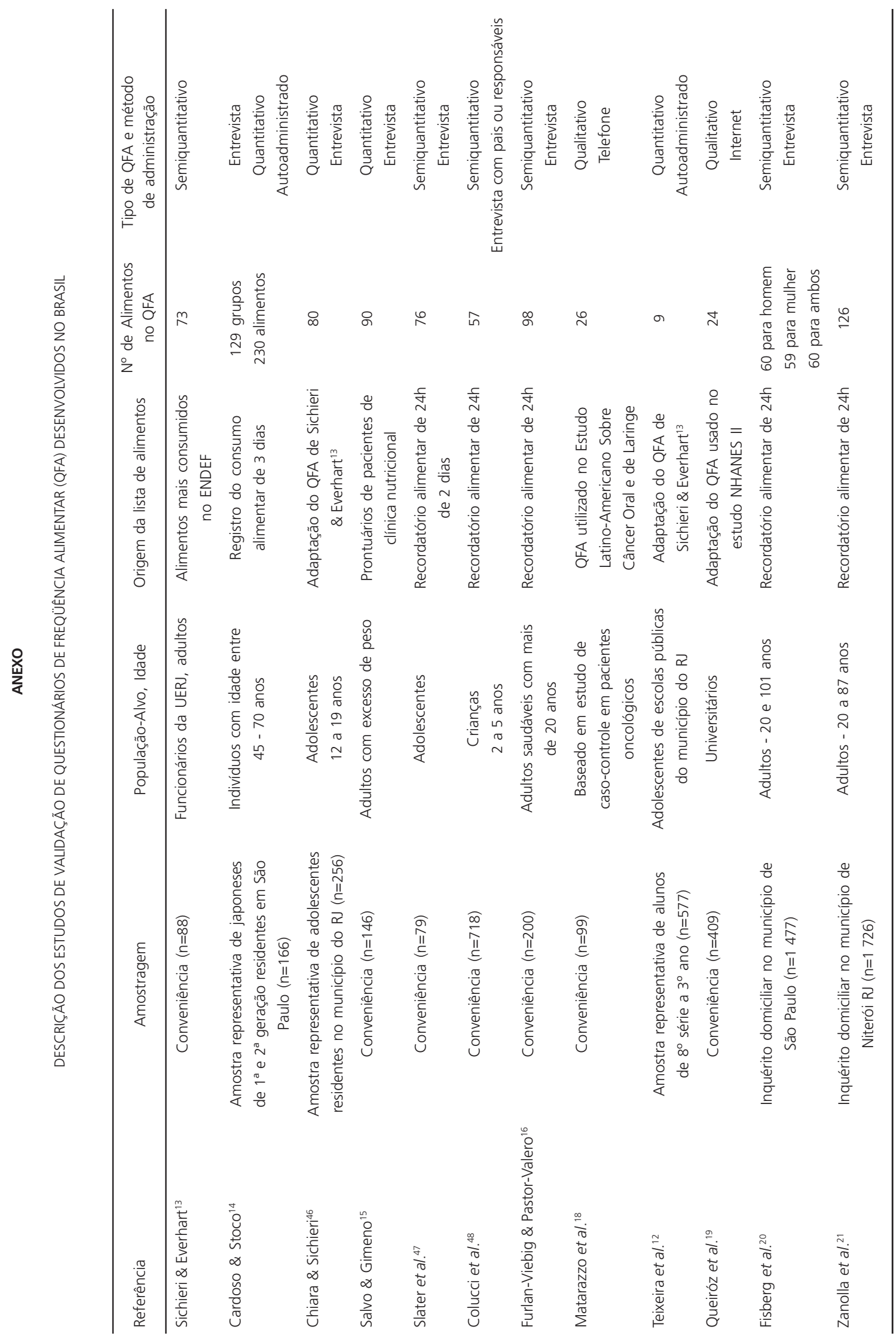


